\title{
Perception of Older People on Dying, Withdrawal of Life Sustaining Intervention and Advance Directives in South Korea
}

\author{
SooOk Kim ${ }^{1}$ and MeeSuk Wang ${ }^{2}$ \\ ${ }^{1}$ Dept. of Nursing, chunnam Techno University, 113, Daehak-ro, Okgwa-myeon, \\ Gokseong-gun, Jeollanam-do, 516-911, Korea \\ ${ }^{2}$ Dept. of Nursing, Hanseo University, 46 Hanseol Ro, Haemi-myun, Seosan-si, \\ Chungcheongnam-do, 369-709, Korea \\ ${ }^{1}$ sokim@chunam-c.ac.kr, ${ }^{2}$ mswang@hanseo.ac.kr
}

\begin{abstract}
The aim of this study was to explore the perceptions of older people on dying, life sustaining interventions, and advance directives (ADs). The questionnaire was consisted of 11 questions regarding demographic characteristics, 5 about perceptions of death and dying, 7 about life sustaining interventions and 6 about Advance Directives (AD). The questionnaire was distributed to 224 participants aged 65 and over with a response rate of 93\%. Data analysis was completed with PASW statistics 18.0 program using the t-test and ANOVA, and Duncan's post hoc test. The majority of people disagreed with that dying is a normal part of life and death should be avoided at all costs. $83.5 \%$ of the participants did not agree with life sustaining interventions. However, 54\% did not know about life sustaining interventions. $72 \%$ $(n=144)$ expressed wishes to participate in decision making on life sustaining interventions for themselves. However, $81 \%(n=162)$ did not know about Advance Directives (AD). Higher awareness of life sustaining intervention correlated with higher awareness level of advance directives ( $r=.253, p<.001)$. There is a need for the development of an educational program tailored at the old people's level of understanding and the construction of social conditions that facilitates acceptance of advance directives as a natural stage in preparing for death, and consequently enable the elderly to spend the remainder of their lives more meaningfully.
\end{abstract}

Keyword: Advance directives, Dying, Elderly, Life sustaining intervention

\section{Introduction}

Advancements in medical technology have not only resolved diseases previously considered incurable, but contributed to extension of life expectancy across the world. The proportion of older people aged 65 years and over among the total population was $7.3 \%$ in 2000 . This is expected to reach $14 \%$ in 2018 and $20 \%$ in 2026.

The dilemma of ageing population and meaningless extension of one's life summoned the issues of bioethics and dying with dignity to the forefront of discussion [1]. Old people experience the most proximity to the issues related to death, life sustaining intervention, and quality of life than any other stages of the lifecycle due to the chronic illnesses. For these reasons, in recent years in South Korea,

Article history:

Received (January 15, 2020), Review Result (February 24, 2020), Accepted (March 29, 2020) 
there has been increasing interest in Advance Directives (ADs). ADs are preparations for the future in pursuit of dying with dignity while they are capable by expressing their will and making provisions for the directions of the treatments they prefer if they become unable to make autonomous decision [2]s. Death is the final stage of one's life, and the acceptance of and the preparation for death are considered as the factors of a good death [3][4]. However, inevitably individuals' beliefs as well as cultural background influences and shapes one's perception of and preparation for death [5][6]. Thus, it is important to understand how the Korean older people perceive dying and death in order to assist them in preparing for and upholding their dignity in death. The aim of this paper is to provide the baseline evidence on the perception of the old people in South Korea on dying, life sustaining intervention and AD.

The aims of this research were to; 1) examine the old people's perceptions of dying, life sustaining intervention and advance directives, and 2) examine the relationship between the subject's characteristics and perceptions of dying, life sustaining intervention and advance directives.

\section{Methods}

\subsection{Study design}

This paper is a descriptive and comparative study. The questionnaire was modified from the North Carolina American Association of Retired Persons (AARP) End-of-Life (EOL) Care Survey [7] (Straw and Cummins 2003). Cronbach's $\alpha$ for the scale of beliefs and values on death, dying, truth telling and ACP was 0.75 , and preferences for life sustaining treatment was 0.71 [8][9] (Johnson, Kuchibhatla and Tulsky 2008). 'Perception of dying' tool in this study provided Cronbach's alpha $=0.598$ and 'preferences for life sustaining treatment' tool gave Cronbach's alpha $=0.855$.

\subsection{Participants}

The participants of this research included 200 old people; 1) who were aged 65 or more, 2) who agreed to partake in the survey, and 3) whose cognitive functions and communicative abilities were not impaired (MMSE 20 or higher). The minimum sample size was calculated using $\mathrm{G}$ power 3.1.2 analysis ware, and was calculated to be 176 when significance level was set at 0.05 , effect size at 0.50 and test power at 0.95 thus the sample size for this study, 200 subjects, should be deemed appropriate.

\subsection{Ethical considerations}

The Researchers were trained in research ethics. Prior to data collection, the participants were made aware of the aim and the procedures of the research. They were assured of the confidentiality and anonymity as well as strict usage of data collected for research purposes only. Also, the participants all voluntarily agreed to participate and were made aware of their right to freely refuse or withdraw participation without any disadvantage.

\subsection{Data collection and analysis}

Three RACFs (residential aged care facilities) were approached after being granted permission from the managers. Old people who were living in their own home were initially approached by visiting nurses in community. It took approximately 20 to 25 minutes to complete each questionnaire. Data collection period for this research was from 5th Dec, 2016 
until 14th May, 2017. The collected data were analyzed via Predictive Analytics Soft Ware (PASW) statistics 18.0. The differences in each participant's perception of dying, life sustaining intervention and advance directives according to the participant's demographic characteristics were analyzed through the t-test and ANOVA, and Duncan's post hoc test was carried out if a noticeable difference was displayed.

\section{Results}

\subsection{Demographic characteristics of participants}

Amongst the 200 subjects, there were 65 males (32.5\%) and 135 females (67.5\%). There largest age group was 65-69 with 84 subjects (42.0\%), and fifty participants reported their highest level of educational attainment to be primary (25.0\%), 59 stated high school $(29.5 \%)$. The place of residence was their own house for 111 subjects (55.5\%) and an aged care facility for $89(44.5 \%)$. For the subjects in home, the majority of 47 lived with their spouse $(42.3 \%)$, 32 lived on their own (28.8\%). The subjects' preferred place of death was their home $(29.5 \%)$ and facility $(70.5 \%)$.

\subsection{Perceptions of dying, and preference for life sustaining interventions and end-of-life care}

The majority of people at home (88.2\%) and RACFs (89.9\%) either disagreed or strongly disagreed with that dying is a normal part of life. Regardless of current residence, the majority of people disagreed or strongly disagreed with that being comfortable (home $86.4 \%$, RACF $87.6 \%$ ) and out of pain (home $82.9 \%$, RACF $87.6 \%$ ) would be more important to me than living as long as possible if they had a disease that could not be cured. Less people $(60.4 \%)$ indicated preference for going to the hospital or the emergency room if they had a disease that could not be cured and if they could be taken care of at home. Whilst $75.2 \%$ $(n=67)$ of people at RACF disagreed or strongly disagreed with that being at home would be more important to me than being in the hospital if they had a disease that could not be cured, however, $83.1 \%(n=74)$ of people at RACF indicated preference for going to the hospital or the emergency room if they had a disease that could not be cured and if they could be taken care of at home.

\subsection{Perception on life sustaining interventions and Advance Directives (ADs)}

Almost a half of participants $(46 \%, \mathrm{n}=92)$ answered they had heard of life sustaining intervention. Among them $30 \%(n=60)$ answered that they had some degree of knowledge about life sustaining intervention, followed by $13 \%(n=26)$ who answered they had heard of it but do not know about it in detail. Only 3\% $(n=6)$ answered that they were well-informed about the procedures. The majority of participants $(83.5 \%, \mathrm{n}=167)$ answered that they do not agree to receiving life sustaining treatment in critical conditions whereas $16.5 \%(n=33)$ stated that they agree.

The majority $(72 \%, \mathrm{n}=144)$ of participants answered that the responsibility of decision makeing regarding life sustaining intervention were mostly 'themselves' followed by their spouses $36(18.0 \%)$, their children $19(9.5 \%)$ and 1 answering their siblings $(0.5 \%)$. In the event that they are unable to make the decision for themselves, the participants preferred to have their spouse $(50 \%, \mathrm{n}=100)$, their children $(44.5 \%, \mathrm{n}=89)$, their guardian $(3 \%, \mathrm{n}=6)$, and their siblings $(1.5 \%, \mathrm{n}=3)$ as their substitute decision maker. 
Among the 200 participants, only 38 (19\%) had previously been heard of ADs. 26 of the 38 participants had accessed the information through the television or the news (13\%), 6 through their friends (3\%), 3 through family members (1.5\%) and doctor and others each had $1(0.5 \%)$. None of the participants answered that they had accessed the information through 'nurse'. Among the 38 who had heard of advance directives none had completed one. 86 out of the 200 participants indicated the intentions of completing advance directives whilst 114 $(57.0 \%)$ stated they had no intentions of signing the document.

Among the 114 who responded that they did not have any intentions of completing advance directives, 62 responded it was because they were unaware of the details of the procedure $(54.4 \%), 31$ replied that their families' opinion was more important than theirs (27.2\%), 9 answered that they found it difficult to raise it with their family (7.9\%) and 5 gave lack of legal validity and 5 responded due to its time consuming nature (4.4\%) [Table 1].

Table 1. Perception on life sustaining interventions and Advance Directives (ADs)(n=200)

\begin{tabular}{|c|c|c|c|c|c|c|}
\hline \multicolumn{2}{|c|}{ Characteristics } & \multirow{2}{*}{$\begin{array}{l}\mathrm{N} \\
84\end{array}$} & \multirow{2}{*}{$\begin{array}{c}\text { Mean(SD) } \\
11.75 \pm 1.43\end{array}$} & \multirow{4}{*}{$\begin{array}{c}\mathrm{t} / \mathrm{F} \\
1.322\end{array}$} & \multirow{4}{*}{$\begin{array}{c}\mathrm{p} \\
0.269\end{array}$} & \multirow{4}{*}{$\begin{array}{c}\text { Duncan's } \\
\text { test }\end{array}$} \\
\hline \multirow{3}{*}{ Age(Years) } & $65-69$ & & & & & \\
\hline & $70-79$ & 62 & $11.52 \pm 1.43$ & & & \\
\hline & $\geq 80$ & 54 & $11.33 \pm 1.66$ & & & \\
\hline \multirow{2}{*}{ Gender } & Male & 65 & $11.45 \pm 1.63$ & \multirow{2}{*}{-.777} & \multirow{2}{*}{0.438} & \\
\hline & Female & 135 & $11.62 \pm 1.43$ & & & \\
\hline \multirow{3}{*}{ Marital status } & Married & 121 & $11.65 \pm 1.49$ & \multirow{3}{*}{1.673} & \multirow{3}{*}{0.174} & \\
\hline & $\begin{array}{l}\text { Widow/ } \\
\text { Widower }\end{array}$ & 76 & $11.38 \pm 1.49$ & & & \\
\hline & Never married & 3 & $11.33 \pm 1.16$ & & & \\
\hline \multirow{5}{*}{ Education } & $\begin{array}{l}\text { No formal } \\
\text { schooling }\end{array}$ & 38 & $11.34 \pm 1.74$ & \multirow{5}{*}{2.286} & \multirow{5}{*}{0.062} & \\
\hline & $\begin{array}{l}\text { Elementary } \\
\text { school }\end{array}$ & 50 & $11.56 \pm 1.25$ & & & \\
\hline & Middle school & 30 & $11.03 \pm 1.13$ & & & \\
\hline & High school & 59 & $11.76 \pm 1.66$ & & & \\
\hline & $\geq$ College & 23 & $12.13 \pm 1.36$ & & & \\
\hline \multirow{2}{*}{ Residence } & Home & 111 & $11.59 \pm 1.56$ & \multirow{2}{*}{.216} & \multirow{2}{*}{0.829} & \\
\hline & Facilities & 89 & $11.54 \pm 1.43$ & & & \\
\hline \multirow{2}{*}{ Religion } & Yes & 123 & $11.62 \pm 1.49$ & \multirow{2}{*}{.630} & \multirow{2}{*}{0.530} & \\
\hline & No & 77 & $11.48 \pm 1.52$ & & & \\
\hline \multirow{5}{*}{$\begin{array}{l}\text { Self-reported health } \\
\text { status }\end{array}$} & Very healthy a & 3 & $12.33 \pm 0.58$ & \multirow{5}{*}{4.043} & \multirow{5}{*}{0.004} & \multirow{5}{*}{$\begin{array}{l}d<e \\
d<b\end{array}$} \\
\hline & Healthy b & 82 & $11.80 \pm 1.49$ & & & \\
\hline & Average c & 3 & $11.33 \pm 0.58$ & & & \\
\hline & Not healthy d & 96 & $11.19 \pm 1.33$ & & & \\
\hline & Very not healthy & 15 & $12.53 \pm 2.13$ & & & \\
\hline Have experience death & Yes & 67 & $11.66 \pm 1.60$ & .676 & 0.500 & \\
\hline
\end{tabular}




\begin{tabular}{|c|c|c|c|c|c|c|}
\hline $\begin{array}{c}\text { of someone close(Less } \\
\text { than 3years) }\end{array}$ & No & 132 & $11.50 \pm 1.45$ & & & \\
\hline $\begin{array}{c}\text { Heard of Knew } \\
\text { about lifesustaining } \\
\text { interventions }\end{array}$ & Yes & 92 & $11.80 \pm 1.44$ & & & \\
\cline { 2 - 7 } & No & 108 & $11.36 \pm 1.53$ & 0.037 & 0.733 & \\
\hline $\begin{array}{c}\text { Willing to write } \\
\text { Advance Directives }\end{array}$ & Yes & 86 & $11.52 \pm 1.56$ & & -.341 & 02 \\
\hline
\end{tabular}

\subsection{The correlation among perception of dying, life sustaining interventions and advance directives}

The results of the participants' preference for life sustaining interventions displayed no apparent changes according to the variables in their demographic characteristics. The comparison of an individual's life sustaining treatment preference in accordance with one's perspective on life sustaining intervention displayed that the participants who agreed to withdrawal of life sustaining intervention had distinctly higher preference level than those opposed to life sustaining intervention $(\mathrm{t}=3.285, \mathrm{p}=.001)$. Moreover, the participants who were willing to complete advance directives displayed a noticeably lower preference level in comparison to the subjects with no intentions of complete advance directives $(t=2.153$, $\mathrm{p}=.033)$

\subsection{The correlation among perception of dying, life sustaining interventions and advance directives}

The correlation among participants' perception of dying, life sustaining interventions and advance directives displays a clear link between the perception of life sustaining intervention and the perception of advancement directives $(r=.253, p<.001)$; higher awareness of life sustaining intervention correlated with higher awareness level of advance directives. However no distinctive differences were marked between perception of dying and life sustaining treatment or between perception of dying and advance directives [Table2].

Table2. The correlation among perception of dying, life sustaining interventions and Advance Directives (ADs) $(n=200)$

\begin{tabular}{|c|c|c|c|}
\hline \multirow{2}{*}{ Variable } & Perception of dying & $\begin{array}{c}\text { Life Sustaining } \\
\text { Interventions }\end{array}$ & $\begin{array}{c}\text { Advance } \\
\text { Directives } \\
\text { (ADs) }\end{array}$ \\
\cline { 2 - 4 } & $\mathrm{R}(\mathrm{p})$ & $\mathrm{R}(\mathrm{p})$ & $\mathrm{R}(\mathrm{p})$ \\
\hline Perception of dying & 1 & & \\
\hline Life Sustaining Interventions & $.125(.080)$ & 1 & 1 \\
\hline Advance Directives (ADs) & $.181(.012)$ & $.253(<.001)$ & 1 \\
\hline
\end{tabular}




\section{Discussion}

In earlier studies, Koreans appeared to escape the initial negative perception of dying [5][10][11] and start developing a more open and positive attitude toward death [12][13]. They found that death is becoming to be accepted as an integral part of life and there was an inverse relationship between the level of health and awareness of death, and the level of awareness also increased as their age increased. However, the results of this study have discovered the old people's perceptions of dying to be less than positive.

The majority of them, regardless of their current residence, indicated that dying is not a normal part of life and that death should be avoided at all costs. Nevertheless, it is promising that almost a half of respondents (home 48.6\%, RACF 44.9\%) felt comfortable talking about death. This is also evident in that desire to exercise one's autonomy in making decisions regarding life sustaining treatment was high at $72 \%$, coinciding with the results of a preceding study that concluded the patients tend to desire to be informed of their prognosis prior to coming to a decision on advance directives [14]. However, in Asian cultures with ties to Confucianism such as Korea, Japan and China family-based decision making is seen as a function of 'filial piety' as opposed to individual self-interest [14]. Thus it is advisable that the family is involved in decision making when old people exercise their right to selfdetermination and that old people's own wishes are reflected in the final decision of composing advance directives.

In previous studies, Korean elderly preferred own home as place of death over hospital[4][6]. Old people desire to complete their life in their own homes where their loved ones are present when they are prepared to find their ego identity and when they are prepared for their deaths [6]. The implications of this for nurses and other health care professionals are obvious in their role to initiate and support their patients to engage in formal and informal discussions with their family members concerning future health care preferences and desires.

The majority of old people in this study disagreed with life sustaining interventions which is similar to the preceding investigations [6][15]. In the Korean society, this trend can be attributed to the stigma attached to life sustaining intervention of being a futile extension of life. However, on the awareness of life sustaining intervention, $30 \%$ had some knowledge of the procedures, and few old people possess a sound understanding or information regarding life sustaining treatments. The low figures were not dissimilar to a preceding study that had examined the old people living in a community[14]. The majority of respondents in this study preferred to have their spouse, son or daughter as their decision maker. This is consistent with the recent study conducted by Jeong et al. (2010). However, one must question if the existing legal, ethical and social framework in South Korea support this practice.

Those who had heard of advance directives was a low number of $19 \%$, similar to the study performed on the old people residing in regional communities and the study performed on middle aged adults [16]. However, $43 \%$ of old people in this study answered that they were willing to compose advance directives. On one hand, it suggests that Korean old people are still very much dependent on external influences such as their family members or the medical professionals in decision making, in addition to the characteristics of a regional community culture [17]. On the other hand, it clearly indicates that there is still a lack of awareness or promotion of advance directives to the Korean old people.

\section{Conclusion}

This study explored the perceptions of old people on dying, life sustaining intervention and advance directives. Old people in this study have demonstrated a particular antagonism 
towards death and a strong obsession with life. They were rather uncomfortable with discussing death and perceiving it as part of life. Nevertheless, the current heightened attention on advance directives as part of one's preparation for death can be seen as a major paradigm shift in Korean sentiments that has negatively marked discussion of death as a taboo. Recently in Korea, the level of national interest on advance directives has surged upwards, yet the old people who are the age group most intimate to the procedures of advance directives have a very low level of awareness or knowledge. Thus there is a need for the development of an educational program aimed at the provision of sufficient information regarding advance directives, tailored at the old people's level of understanding and the construction of social conditions that facilitates acceptance of advance directives as a natural stage in preparing for death, and consequently enable the elderly to spend the remainder of their lives more meaningfully. The study provides nurses in various settings with the baseline evidence and great potential to improve EOL care and ultimately to help older people to 'die well'. The samples for this research were the old people residing in certain regions of Korea, and therefore there are limitations in generalizing the findings of this paper.

\section{References}

[1]. S. Jeong, I. Higgins, and M. McMillan, "The essentials of advance care planning for end-of-life care for older people,” Journal Clinical Nursing, vol.19, no.3, pp.389-397, (2010)

[2]. J. W. Park and J. A. Song, "Knowledge, experience and preference on advance directives among community and facility dwelling elderly," Journal of the Korean Geriatrics Society, vol.33, no.3, pp.581-600, (2013)

[3]. M. S. An and K. J. Lee, "Awareness of good death and attitudes toward terminal care among geriatric hospital nurses," Korean Journal of Hospice and Palliative Care, vol.17, no.3, pp.122-33, (2014)

[4]. M. S. Lee and Y. J. Kim, "Good death recognized by the elderly," Journal of the Korea Contents Association, vol.13, no.6, pp.283-299, (2013)

[5]. J. S. Choi, "Introduction death studies," Seoul; Mosinun Saramdeel, (2013)

[6]. M. S. Kim, "Perception and attitude of Koreans toward death and it's philosophical implication," J New Korean Philosophical Association, vol.64, no.2, pp.43-69, (2011)

[7]. G. Straw and R. Cummins, "AARP North Carolina End of Life Care Survey," AARP Knowledge management, Washington, DC, (2003)

[8]. K. Johnson, M. Kuchibhatla, and J. A. Tulsky, "Racial differences in self-reported exposure to information about hospice care,” Jurnal of Palliative Medicine, vol.12, no.10, pp.921-927, (2009)

[9]. K. Johnson, M. Kuchibhatla, and J. A. Tulsky, "What explains racial differences in the use of advance directives and attitudes towards hospice?" Journal of the American Geriatrics Society, vol.56, no.10, pp.1953-1958, (2008)

[10]. J. S. Choi, "Preparing for death really is not in the bucket list," Won-Buddhist Thought \& Religious Culture, vol.46, pp.1-31, (2011)

[11]. M. S. Kim, M. H. Gang, and Y. O. Kim, "Completion and related factors of advance directives in old adults," Journal of the Korea Contents Association, vol.8, no.2, pp.240-247, (2018)

[12]. Y.G. Lee, P.H. Kim, M.R. Park, and N. J. Je, "Effects of well-dying program on the death anxiety," Perception of Good Death, and Readiness for Death, Journal of the Korea Academia-Industrial cooperation Society, vol.8, pp.522, (2019)

[13]. K. H. Jo, "The meaning of dignified dying perceived by nursing students," J Korean Acad Soc Nurs Educ, vol.16, pp.72-82, (2010)

[14]. H. S. Kim, S. M. Kim, S. J. Yu, and M. Kim., "Preference for care near the end of life of Korean nurses. Korean Journal of Hospice and Palliative Care, vol.13, no.1, pp.41-49, (2010) 
Perception of Older People on Dying, Withdrawal of Life Sustaining Intervention and Advance Directives in South Korea

[15]. J. S. Kim, "The value in quality of life and recognition and preference for Advance Directives by the elderly's age," Journal of the Korean Geriatrics Society, vol.32, no.1, pp.1-23, (2012)

[16]. E. H. Park and N. Y. Kim, "The influence of Nursing Professionalism, Attitudes toward Advance Directive, and Death Anxiety on Terminal Care Performance of Nurses in Long-term Care Hospitals," Korean Journal of Adult Nursing, vol.30, no.2, pp.183-193, (2018)

[17]. S. M. Kim, K. S. Kim, I. S. Lee, and S. H. Kim, "Perception towards advance directives, hospice palliative care, life-sustaining treatment among the middle aged adults," Korean Academy of Social Welfare, vol.37, pp.22-44, (2013) 